\title{
Covariance Matrix Adaptation Pareto Archived Evolution Strategy with Hypervolume-sorted Adaptive Grid Algorithm
}

\author{
Shahin Rostami ${ }^{\mathrm{a}}$ and Ferrante Neri ${ }^{\mathrm{b}, 1}$ \\ ${ }^{a}$ Department of Computing and Informatics, University of Bournemouth, Bournemouth, United Kingdom \\ ${ }^{\mathrm{b}}$ School of Computer Science and Engineering, De Montfort University, Leicester, United Kingdom
}

\begin{abstract}
Real-world problems often involve the optimisation of multiple conflicting objectives. These problems, referred to as multi-objective optimisation problems, are especially challenging when more than three objectives are considered simultaneously.

This paper proposes an algorithm to address this class of problems. The proposed algorithm is an evolutionary algorithm based on an evolution strategy framework, and more specifically, on the Covariance Matrix Adaptation Pareto Archived Evolution Strategy (CMA-PAES). A novel selection mechanism is introduced and integrated within the framework. This selection mechanism makes use of an adaptive grid to perform a local approximation of the hypervolume indicator which is then used as a selection criterion. The proposed implementation, named Covariance Matrix Adaptation Pareto Archived Evolution Strategy with Hypervolume-sorted Adaptive Grid Algorithm (CMA-PAES-HAGA), overcomes the limitation of CMA-PAES in handling more than two objectives and displays a remarkably good performance on a scalable test suite in five, seven, and ten-objective problems. The performance of CMA-PAES-HAGA has been compared with that of a competition winning meta-heuristic, representing the state-of-the-art in this sub-field of multi-objective optimisation.

The proposed algorithm has been tested in a seven-objective real-world application, i.e. the design of an aircraft lateral control system. In this optimisation problem, CMA-PAES-HAGA greatly outperformed its competitors.
\end{abstract}

Keywords. multi-objective optimisation, many-objective optimisation, evolution strategy, selection mechanisms, approximation methods

\section{Introduction}

Optimisation is a theoretical fundamental concept in computational intelligence $[62,63]$ and engineering $[49,77]$. For example, design engineering problems are intrinsically optimisation problems, see e.g. the design of steel structures $[53,54,74]$. Engineering modelling $[69,75,84]$ as well as parameter identification [5] are optimisation problems. Optimisation examples are also in the rail industry $[59,101]$, routing problems [12], and time-cost tradeoff analysis [57].

Many real-world optimisation problems in academia $[35,78,94]$ and industry $[1,47,66]$, such as civil engineering $[29,76]$, often involve the simultaneous optimisation of multiple conflicting objectives, see e.g. $[7,45,87]$.

\footnotetext{
${ }^{1}$ Corresponding Author: School of Computer Science and Engineering, De Montfort University, The Gateway House, LE1 9BH, Leicester, United Kingdom, E-mail: fneri@dmu.ac.uk.
}

These problems are known as multi-objective optimisation problems, see e.g. [20,65], and their goal consists of finding a set of solutions which cannot be outperformed over all of the considered objectives (i.e. the solutions do not dominate each other) $[9,16]$. This set, namely the Pareto set, is often a theoretical abstraction and either cannot be found in practice or may have, on its own, no immediate practical use. The first case occurs when the objective functions are defined on a dense set. Under this condition the Pareto set would be composed of an infinite number of elements, which cannot be computed. The second case occurs in applications where one (or at most a few) answers to the optimisation problem must be given. This is often the case in engineering design where ultimately one solution must be chosen, see e.g. [1,7,73].

Unless specific hypotheses allow an exact approach, a good multi-objective optimisation algorithm works on a set of candidate solutions, often referred 
to as a population, to detect a discrete approximation of the Pareto set also referred to as an approximation set. This statement is true for diverse frameworks such as Genetic [20,21] and Evolutionary Algorithms [81,98], local search [56], Differential Evolution [64,93], Memetic Frameworks [42,79] in both theoretical and application domains [55]

This approximation is a set of candidate solutions that represent the theoretical Pareto set. The features that describe the quality of an approximated Pareto set have been theorised in [67]. These features are:

- Proximity: The detected approximated Pareto set is desired to be as close as possible to the true Pareto-optimal front. Unfortunately, proximity cannot be used as a measure of quality of the approximation set during the optimisation process, because the true Pareto set is not known (otherwise the problem would already be solved).

- Diversity: The desired approximation set should be uniformly distributed across the trade-off surface of the problem. This characterises the distribution of the approximation set both in the extent and uniformity of that distribution.

- Pertinence: Ideally, the approximation set should contain a number of solutions which are within some area (namely region of interest, see below) of the objective space. With this feature, the selection of a candidate solution from an approximation set becomes less complicated.

A representation of the desired features of an approximation set are graphically represented in the twoobjective case in Fig. 1, see [72].

With reference to the Pertinence of an approximation set, the process of performing the selection of the solution among the available candidate solutions is named Decision Making. The criterion or algorithm that leads to the decision making is said to be the Decision Maker (DM). In other words, the DM implicitly classifies 'interesting and uninteresting' solutions. The area of the objective space where the interesting solutions fall within is named the Region Of Interest (ROI).

In summary, the solution of a multi-objective optimisation problem can be interpreted as a twostage process: during the first stage the multi-objective

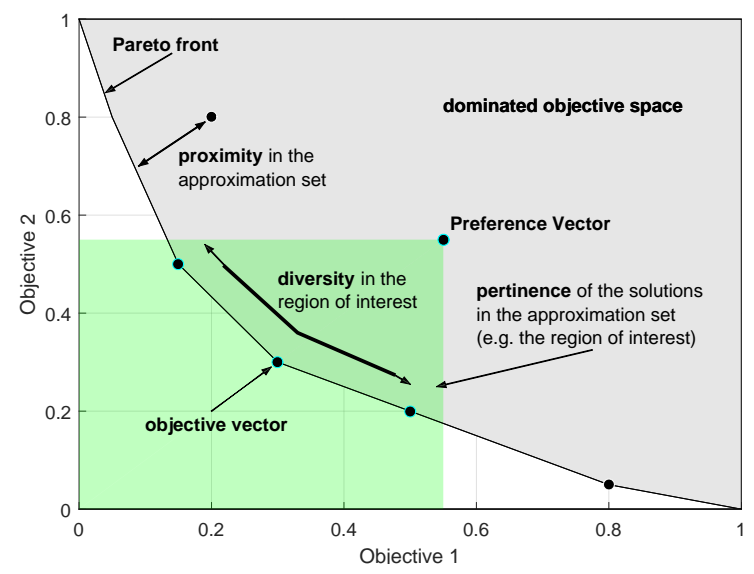

Figure 1. Proximity, diversity, and pertinence characteristics in an approximation set in two-objective space.

optimisation algorithm detects the approximation set while the DM performs the selection of the interesting solution from the approximation set in the second stage.

During both the stages, the main difficulty of the problem is the comparison and selection of solutions. Since one solution can outperform another solution with respect to one objective and not with respect to another (the solutions do not dominate each other), the selection of a set of solutions from a larger set is a challenging task. This task becomes progressively more challenging as the number of objectives increases, see [25].

\subsection{Multi-objective optimisation with more than three objectives: related work}

The objectives of a multi-objective problem span a multi-dimensional space, namely the objective space. The size of the objective space grows exponentially with the number of objectives. This is analogous to the effect of dimensionality on the search space: for example a problem with 1000 variables is much harder (and not just twice) than a problem in 500 variables. A high-dimensional objective space is very hard to handle when a pairwise comparison is performed. Thus, the difficulty of a multi-objective problem grows exponentially with the dimensionality of the objective space.

A multi-objective optimisation problem with more than three objectives is often referred to as a many- 
objective optimisation problem $[25,40,58]$. The study of this class of problem as well as the algorithms designed to tackle the difficulties dictated by a highdimensional objective space are emerging research trends in literature. The main challenges have been highlighted in several articles, see e.g. $[13,14,40]$. These difficulties are detailed in the following list:

- The difficulty of performing pairwise comparisons with the aim of selecting a set (a new population) increases with the number of objectives, as it is likely that almost all candidate solutions within a population do not dominate each other, see $[27,52,68]$.

- As a consequence of the dimensionality of the objective space, the number of candidate solutions required to produce an approximation set increases exponentially with the number of objectives, see $[37,46]$.

- With the increase in the number of objectives, the computational cost of existing search operators becomes infeasible [37].

- When the number of objectives increase, the number of generations required to produce an approximation set also increases exponentially.

- The increase in the number of objectives makes the problem less intuitive. Approaches which rely on spatial information between solutions in the objective space become ineffective. Furthermore, the visualisation of candidate solutions becomes difficult, often resulting in the use of heat-maps or parallel-coordinate plots. This poses a difficulty to the DM as the selection of a final candidate solution may become non-intuitive [86].

As a consequence, popular algorithms that perform well on multi-objective optimisation with two or three objectives $[15,20,82]$ display poor performance when applied to a problem that has many objectives, e.g. five, see $[33,34,39]$. Studies on specific problems confirmed this fact $[2,44]$ while other articles have observed this phenomenon with the aim of proposing methods for tackling a high number of objectives $[48,68,103]$ such as an objective space reduction by the inclusion of the preferences within the search [61].

Therefore, several alternative algorithmic solutions have been proposed to tackle the difficulties posed by a large number of objectives. An interesting example is the Non-dominated Sorted Genetic Algorithm III (NSGA-III), see [19,43] based on the previous NSGA-II. In this case, the dominance selection is revisited and adapted to the many-objective case by the use of structured weights (reference-points), in order to maintain high diversity throughout the optimisation process. This approach is similar to that taken in the Multi-Objective Evolutionary Algorithm based on Decomposition (MOEA/D) introduced in [95] to address the same difficulty, such that both NSGA-III and MOEA/D can be initialised with the same set of weights. Another feature of NSGA-III and MOEA/D is the use of niching during the selection of parent solutions for the recombination stage. This mechanism has been proposed to increase the exploitation of the algorithm. A further development of the MOEA/D idea has been presented in [96] where the MOAE/D with Dynamic Resource Allocation (MOEA/D-DRA) has been introduced. This MOEA/D-DRA decomposes the many-objective space into many singleobjective spaces (sub-problems) and then assigns different computational budgets to the various subproblems. This algorithm, which has been a competition winner at IEEE CEC [97] is currently one of the most effective solutions to tackle many-objective problems.

In [99] an acceleration of the search is achieved by biasing it: the direction of the knee points of the Pareto set (those points which represent the lack of a clear preference towards a specific objective) is preferred. This approach shares with [98] the philosophy of avoiding extensive pairwise comparisons and handling large objective spaces better. Furthermore, this approach can be seen as a modern re-interpretation of a weighted sum approach where each weight coefficient is set to 1 .

In $[10,11]$ the reference vector, i.e. a vector containing the preference weights for each objective, is integrated within the selection process in order to exclude solutions which do not fall within the ROI from the search. The idea of the incorporation of DM preferences into the algorithmic selection has been proposed in other contexts, e.g. [60,70,71].

Over the past decade, the use of some metrics to measure the quality of non-dominated solution sets, namely performance indicators, have been introduced 
to effectively perform the selection of solutions in the presence of many objectives. For example, the Indicator Based Evolutionary Algorithms (IBEAs) [41, 100, 102] use performance indicators as a method of introducing selection pressure in place of dominance based selection, such as [21]. Amongst these indicators the hypervolume indicator, see e.g. [28], is a very powerful approach which can lead to high performance results.

The hypervolume indicator measures how much of the objective space is dominated by an approximation set. The way the hypervolume indicator is constructed is very appealing in real-world problems as it requires no information regarding the theoretical Pareto-front (which is often unknown in practise.). Furthermore, the hypervolume indicator encompasses within it the information about proximity, diversity, and pertinence, ultimately evaluating the quality of the approximation set, see [31]. Successful examples of incorporating the hypervolume indicator into the optimisation process during the selection stage can be found in [22] and [3], where an adapted version of the hypervolume indicator, named the contributing hypervolume indicator, is used. Other successful cases of the hypervolume indicator being used during the selection process are given in [36] and [85] within the context of the Multi-Objective Covariance Matrix Adaptive Evolution Strategy (MO-CMA-ES).

Although hypervolume indicator is a very valuable sorting criterion for selection, its calculation presents the drawback that it depends exponentially on number of objectives and becomes infeasible in manyobjective problems. For this reason several papers attempted to propose algorithms for a fast calculation of the hypervolume indicator.

In [91] an algorithm based on a set theory of union and intersection of sets is proposed to calculate the hypervolume. The method proposed in [26] calculates the hypervolume as the sum of contribution given by the volume dominated by each solution, one by one. The latter method has been used to perform selection within an Evolutionary Multi-Objective (EMO) algorithm in [50] which still resulted in being computationally onerous. Several approximated calculations for the hypervolume indicator have been proposed, see e.g. [24]. In [88], the complexity of the hypervolume indicator is reduced by considering the objectives one by one. In [3], the hypervolume indicator is approximated by means of a Monte Carlo simulator.

\subsection{Proposal of this article}

While selection processes based on hypervolume indicators can be integrated in all meta-heuristics, not all the frameworks would be equally suitable to their embedding and their simplified calculation. Based on this consideration, this paper proposes a novel metaheuristic for multi-objective optimisation which integrates a novel hypervolume indicator based selection process within the Pareto Archived Evolution Strategy (PAES) [51]. The latter is an algorithm that performs the selection of the individuals by grouping the solutions into sub-regions of the objective space. This mechanism is named the Adaptive Grid Algorithm (AGA). The resulting algorithm, namely the Covariance Matrix Adaptation Pareto Archived Evolution Strategy with Hypervolume-sorted Adaptive Grid Algorithm (CMA-PAES-HAGA) makes use of the search logic of the Covariance Matrix Adaptation Evolution Strategy (CMAES) [30] and the archive of the PAES structure. A preliminary version of the proposed algorithm without the newly proposed selection mechanism has been proposed in [72], however, its primitive selection mechanism restricts its application to two-objective problems only.

CMA-PAES-HAGA proposes an algorithm for multi-objective optimisation which is specifically suited for four or more objectives.

The remainder of this article is organised in the following way. Section 2 introduces the notation and describes the proposed CMA-PAES-HAGA. Furthermore, Section 2 provides the reader with the motivation behind CMA-PAES-HAGA and emphasizes the differences with respect to its predecessor. Section 3 tests the proposed algorithm and compares it against the state-of-the-art and competition winning algorithm MOEA/D-DRA. This comparison is carried out over a set of test problems and over a real-world multiobjective problem with seven objectives concerning an aircraft control system. Finally, Section 4 presents the conclusion to this work. 


\section{Covariance Matrix Adaptation Pareto Archived Evolution Strategy with Hypervolume-sorted Adaptive Grid Algorithm}

Covariance Matrix Adaptation (CMA) is a powerful approach to parameter variation which has demonstrated promising results in the optimisation of singleobjective problems with CMA-ES [36], and multiobjective problems with MO-CMA-ES [85] and CMAPAES [72]. These optimisation algorithms rely on CMA entirely for the variation of solution parameters, and therefore they do not suffer from the curse of dimensionality which affects many optimisation algorithms which rely on reproduction operators. CMAPAES is a multi-objective evolution strategy which first incorporated the CMA approach to variation with an adaptive grid scheme and Pareto archive. However, when moving to the optimisation of problems consisting of many (greater than three) objectives, it is the selection operators employed by these algorithms which render them ineffective or computationally infeasible. The algorithm which is introduced in this section, named CMA-PAES-HAGA, proposes an approach to optimisation which is suitable for problems consisting of many objectives.

The approach to selection employed by MOCMA-ES relies on the Contributing Hypervolume Indicator (CHV) as a second-level sorting criterion, the calculation of which increases significantly with the number of objectives considered. In contrast, the approach to selection employed by CMA-PAES is driven by an AGA which incurs little algorithm overhead in its calculation [72]. This AGA becomes ineffective when applied to many-objective problems as the number of grid divisions which controls the division of the objective space and the size of the sub-populations becomes increasingly sensitive with each additional objective [70].

Whilst CMA-PAES has shown promising results in comparison to PAES, NSGA-II, and MO-CMAES [70,72], it suffers from the curse of dimensionality when moving to many-objective problems.

This is because the process of the AGA for diversity preservation was designed with the two-objective case in mind. The grid location was stored as a scalar value starting at one, meaning it was not possible to use auxiliary information e.g. how close one maxi- mally populated grid location was to another. This information is beneficial when deciding which grid population, from many which are maximally populated, will be selected for solution replacement when the archive is at capacity. The original AGA in CMAPAES also replaced a solution at random, this approach does not cause a significantly negative impact on its performance in the two-objective case, when configured with the recommended high number of grid divisions. However, when moving to problems consisting of a high number of objectives, a smaller number of grid divisions must be selected, and a more sophisticated approach to solution replacement must be considered.

CMA-PAES-HAGA is an Evolutionary Multiobjective Optimisation (EMO) algorithm, but more specifically, it is an evolution strategy for solving optimisation problems consisting of many objectives. CMA-PAES-HAGA achieves many-objective optimisation by: 1) employing CMA for variation and opting out of the use of reproduction operators; 2) employing a new approach to selection in the form of a hypervolume-sorted AGA.

The following section begins by introducing the notation used throughout the paper, followed by a description of the CMA-PAES-HAGA algorithm including pseudo-code, mathematical procedures, and worked-through examples of the operators. The section concludes by defining the variants of CMAPAES-HAGA and how they are implemented.

\subsection{Notation and Data Structures}

With the aim of defining the notation and datastructures used throughout this paper, $M$ defines the number of objectives, $N$ defines the population size, and a solution is defined by the tuple:

$$
\left[X_{n}, V_{n}, \bar{p}_{s u c c, n}, \sigma_{n}, \sigma_{n}{ }^{*}, p_{n, c}, \mathbf{C}_{n}\right]
$$

where $X_{n}$ and $V_{n}$ are pointers to a solution's objective values and decision variables respectively. $X$ is an $M$ by $N$ matrix of the entries $x_{m n}$, where every entry $x_{m n}$ refers to a solution's objective value: 


$$
\mathrm{X}_{\mathrm{n}}=\left\langle x_{1 n}, x_{2 n}, \ldots, x_{M n}\right\rangle
$$

Similarly, $V$ is an $I$ by $N$ matrix of the entries $v_{i n}$, where every entry $v_{i n}$ refers to a solution's decision variable:

$$
\mathrm{V}_{\mathrm{n}}=\left\langle v_{1 n}, v_{2 n}, \ldots, v_{I n}\right\rangle
$$

An objective function is required to evaluate the performance of candidate solutions (i.e. those solutions that could be the optimum). There can exist $M$ objective functions with the definition in (4), these objective functions can be either minimised or maximised.

$$
f\left(V_{n}\right)=\left(f_{1}(v), f_{2}(v), f_{3}(v), \ldots, f_{M}(v)\right)
$$

A multi-objective optimisation problem in its general form can be described as:

$$
\left.\begin{array}{lll}
\text { optimise } & f_{m}(v), & m=1,2, \ldots, M ; \\
\text { subject to } & g_{j}(v) \geq 0, & j=1,2, \ldots, J ; \\
& h_{k}(v)=0, & k=1,2, \ldots, K ; \\
& v_{i}^{(L)} \leq v_{i} \leq v_{i}^{(U)} & i=1,2, \ldots, I ;
\end{array}\right\}
$$

The constraint functions $g_{j}(v)$ and $h_{k}(v)$ impose inequality and equality constraints that must be satisfied by a solution $v$ in order for it to be considered a feasible solution. Another condition which affects the feasibility of a solution, regards the adherence of a solution $v$ to values between the lower $v_{i}^{(L)}$ and upper $v_{i}^{(U)}$ boundaries. The set containing all the feasible solutions is referred to as the decision space. The corresponding set of the values that each feasible solution can take is referred to as the objective space.

Each solution tuple also includes parameters used by the CMA variation operator, where $\bar{p}_{\text {succ }, n} \in[0,1]$ is the smoothed success probability, $\sigma_{n} \in \mathrm{R}_{0}^{+}$is the global step size, $\sigma_{n}$ is the previous generation's global step size, $p_{n, c} \in \mathrm{R}^{n}$ is the cumulative evolution path, and $\mathbf{C}_{n} \in \mathrm{R}^{v \times v}$ is the covariance matrix of the search distribution.

\subsection{Algorithm design}

The CMA-PAES-HAGA process outlined in Algorithm 1 begins by initialising algorithm parameters and randomly sampling the search-space to generate an initial parent population $X$ of size $\mu$, the objective values of each parent solution are then resolved using an objective function. $X$ is an $M$ by $N$ matrix of entries $x_{m n}$, where every $x_{m n}$ refers to a solution's objective value, and $X_{n}$ refers to a solution (3).

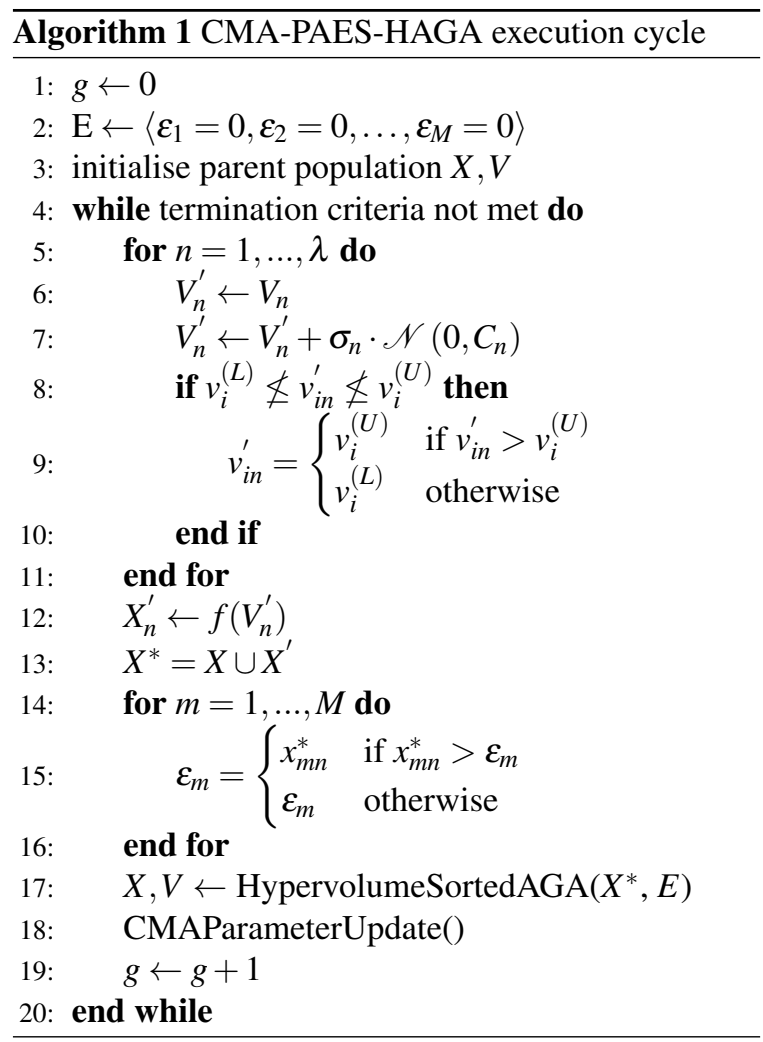

The global CMA parameters are: the target success probability $p_{\text {succ }}^{\text {target }}$, success threshold $p_{\text {thresh }}$, step size damping $d$, step size learning rate $c_{p}$, evolution path learning rate $c_{c}$, and the covariance matrix learning rate $c_{c o v}$. The values for these parameters are set according to the parameters proposed in [36] where $p_{\text {succ }}^{\text {target }}=(5+\sqrt{1 / 2})^{-1}, p_{\text {thresh }}=0.44, d=1+n / 2$, $c_{p}=p_{\text {succ }}^{\text {target }} /\left(2+p_{\text {succ }}^{\text {target }}\right), c_{c}=2 /(n+2)$, and $c_{\text {cov }}=$ $2 /\left(n^{2}+6\right)$.

With the initial parent population ready, the generational loop begins: 


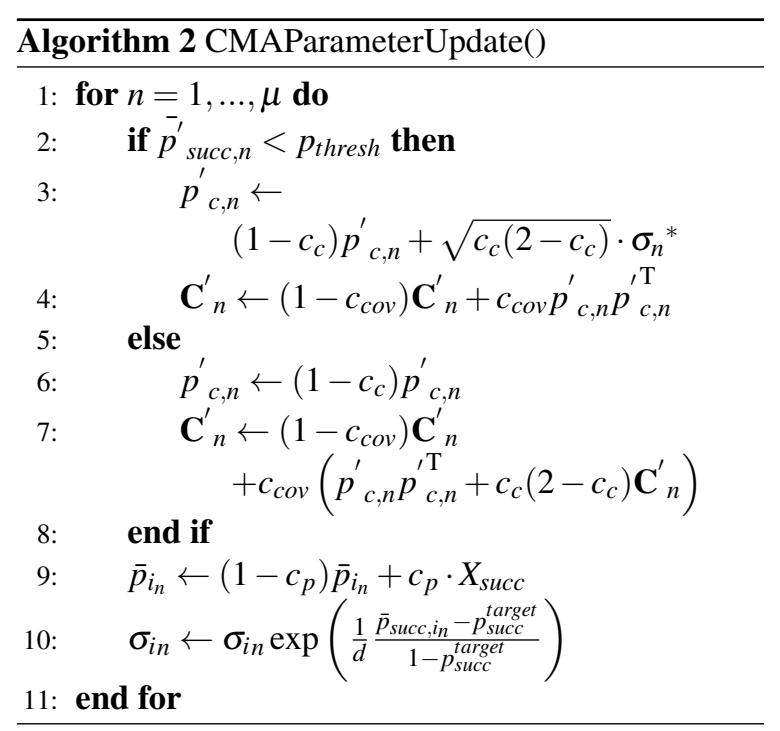

- The termination criterion is checked to decide whether the EMO process is terminated. In CMA-PAES-HAGA, the default condition for termination depends on reaching a maximum number of function evaluations (specified a priori).

- The offspring population $X^{\prime}$ of size $\lambda$ is then generated using the parent population $X$ and the CMA operator for variance.

- The offspring population's solutions are then evaluated using an objective function, this population is then merged with the parent population to create the intermediate population $X^{*}=X \cup X^{\prime}$.

- The extreme values $\varepsilon_{m}$ encountered for each objective during the optimisation process are then updated by checking if any objective value $x_{m n}^{*}$ is higher than a corresponding stored extreme objective value $\varepsilon_{m}$, and if so, replacing it.

$$
\varepsilon_{m}= \begin{cases}x_{m n}^{*} & \text { if } x_{m n}^{*}>\varepsilon_{m} \\ \varepsilon_{m} & \text { otherwise }\end{cases}
$$

where $E$ is a vector containing all of the extreme values encountered for each objective.

$$
\mathrm{E}=\left\langle\varepsilon_{1}, \varepsilon_{2}, \ldots, \varepsilon_{M}\right\rangle
$$

- The intermediate population $X^{*}$ is then subjected to the hypervolume-sorted AGA selection mechanism described in Section 2.3. The mechanism will return a new parent population of size $\mu$ which are considered to offer the best coverage of the objective space.

- The parameters used for the CMA operator for variance are then updated according to Algorithm 2 . The solutions are considered successful (and marked as $X_{\text {succ }}=1$ ) if they make it from the intermediate population $X^{*}$ to the parent population for the next generation. Conversely, the solutions are considered unsuccessful (and marked as $X_{\text {succ }}=0$ ) if they are not transferred to the following generation and are not retained.

- The optimisation process then continues to the next generational iteration.

\subsection{Hypervolume-sorted Adaptive Grid Algorithm}

CMA-PAES-HAGA employs the Hypervolume-sorted AGA (HAGA) to select solutions to form the parent population for the next generation of the optimisation process. HAGA is a two-phase approach to selection, with the aim of being computationally feasible in the presence of many objectives. HAGA incorporates the use of a novel AGA implementation containing a number of features in order to make the AGA implementation suitable for many-objective optimisation. These features consist of:

- A new data structure for storing a solution's grid number up to any number of objectives;

- A new grid-proximity method for grid selection when searching for a solution to remove;

- A new scheme for the maintenance of global extremes for objectives.

HAGA aims to use a two-phase approach to reduce the number of solutions which are to be considered by the narrow-phase, through the use of a broad-phase. The grid structure when visualised for a two-objective approimation set can be seen in Figure 2, where the grid squares have been indicated with dashed lines, and the grid locations have been indicated with vectors surrounded by braces. In this example, the grid location $\{2,3\}$ refers to a grid square 
which defines a grid population consisting of the solutions $X_{3}, X_{4}$ and $X_{5}$.

This is achieved by mean of the execution lifecycle listed in Algorithm 3, where $X$ is an approximation set of solutions $X_{n}, A$ is the archive of parent solutions selected by HAGA, $\Gamma$ is a grid location consisting of multiple solutions, and $\mu$ and $\lambda$ are the number of parent and offspring solutions respectively. $C H V_{\Gamma_{t}}$ is a vector of entries resulting from the execution of the contributing hypervolume indicator on the solutions in the grid population $\Gamma_{t}$, such that $\min \left(C H V_{\Gamma_{t}}\right)$ would yield the solution which offers the lowest explicit hypervolume indicator contribution in regards to the observed grid population.

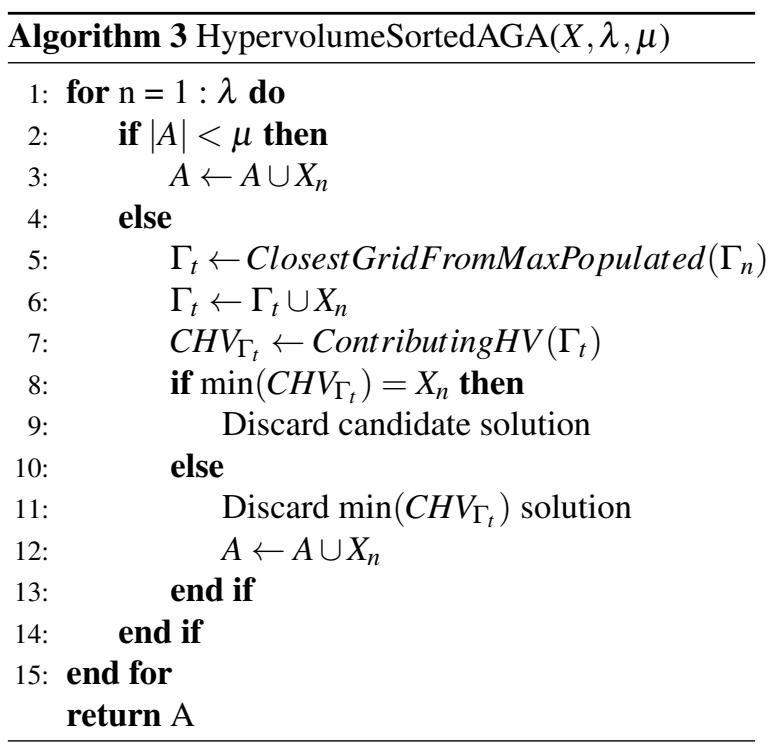

In Algorithm 3, Line 5 identifies the grid location closest to the candidate solution and resolves a grid population. This is considered the broad-phase of the two-phase approach. Once a grid location has been identified, it is used in the calculation of the narrowphase (Line 7), which depends on the contributing hypervolume indicator described in the following.

The basic principle of HAGA is to benefit from the CHV algorithm's ability to discriminate solutions based on the explicit hypervolume they contribute to a population, but to do so in a way that doesn't introduce the computational infeasibility of using CHV on populations consisting of many-objective solutions. HAGA achieves this through the use of the adaptive grid, where the objective space covered by a population is divided into a grid consisting of grid areas. This grid has a capacity for the number of solutions it can store, this capacity is set to $\mu$, which is the number of parent solutions desired for selection. The solutions within the intermediate population (the parent and offspring population) are then added to this grid one by one. Throughout this process, there is no computation of the CHV algorithm until the grid reaches capacity, at which point the $\mathrm{CHV}$ is only computed at grid area level. This ensures that the $\mathrm{CHV}$ is computed for only a small number of solutions, in order to determine which solution is to be evicted from the grid area to prevent the grid from exceeding capacity. In contrast, the CHV algorithm simply computes the CHV indicator value for every solution in the population.

The CHV indicator can be calculated by first calculating the hypervolume indicator quality $X_{H V}$ of a population $X$ :

$$
\begin{aligned}
& H V\left(f^{r e f}, X\right)= \\
& \Lambda\left(\bigcup_{X_{n} \in X}\left[f_{1}\left(X_{n}\right), f_{1}^{r e f}\right] \times \cdots \times\left[f_{m}\left(X_{n}\right), f_{m}^{r e f}\right]\right)
\end{aligned}
$$

where $f_{m}^{r e f}$ is the reference point for objective $m$, and $X_{m}$ is a set of objective values for objective $m$ from the current population.

With the hypervolume indicator quality of the population calculated, it is possible to calculate the $\mathrm{CHV}$ indicator value of each candidate solution within the population. For each solution in the population, the solution is first removed from the population to form the temporary population $X^{T}$, and then the hypervolume indicator quality $X_{H V}^{T}$ is calculated for this temporary population. The $\mathrm{CHV}$ indicator value is then calculated by subtracting $X_{H V}^{T}$ from $X^{T}$, this is the explicit hypervolume contribution of the candidate solution. Once the CHV indicator value has been calculated for every candidate solution, it is possible to order them by descending value so that they are ordered by the greatest explicit hypervolume indicator contribution. The first $\mu$ solutions are then selected to form the next parent population. This approach has been listed in Algorithm 4, where $X$ is a set of solutions from a grid population. 


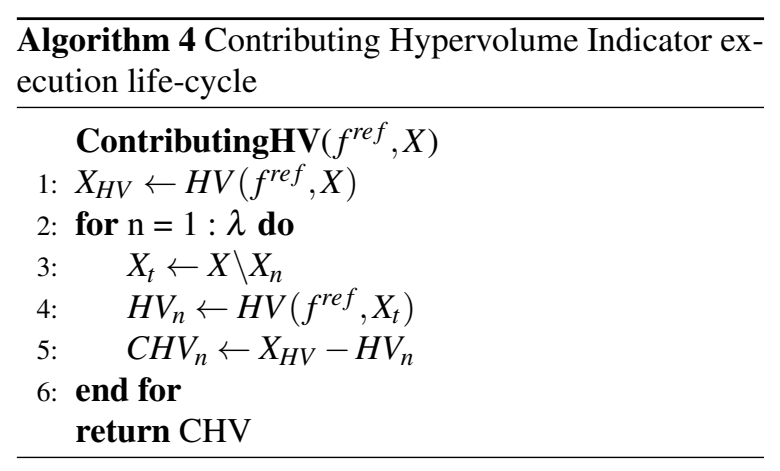

It must be noted that the purpose of HAGA is to return a set of solutions which is of size $\mu$, this size parameter must be defined before the execution of the algorithm. The $\mu$ size parameter is used within the HAGA selection approach, and as such the output set is dependent on its definition. This is unlike the CHV selection approach, which will return a set of hypervolume indicator values for each solution in the set on which it was executed.

The process for HAGA in its entirety is mathematically described herein. $\Delta$ defines the number of desired grid divisions for an objective within the objective space. $\Gamma$ is an $M$ by $N$ matrix of entries $\gamma_{m n}$,

$$
\Gamma_{n}=\left\langle\gamma_{1 n}, \gamma_{2 n}, \ldots, \gamma_{M n}\right\rangle
$$

where $\Gamma_{n}$ refers to a row in the $\Gamma$ matrix, and every entry $\gamma_{m n}$ refers to the grid location of an objective value $x_{m n}$ in the divided objective space.

To calculate $\Gamma_{n}$, the grid location $\gamma_{m n}$ of each objective value $x_{m n}$ for each solution $X_{n}$ needs to be resolved. To calculate a solution's grid location, the padded grid length $\Lambda$

$$
\Lambda=\left\langle\lambda_{1}, \lambda_{2}, \ldots, \lambda_{M}\right\rangle
$$

for each objective needs to be calculated using the lowest and highest objective value for each objective in the population:

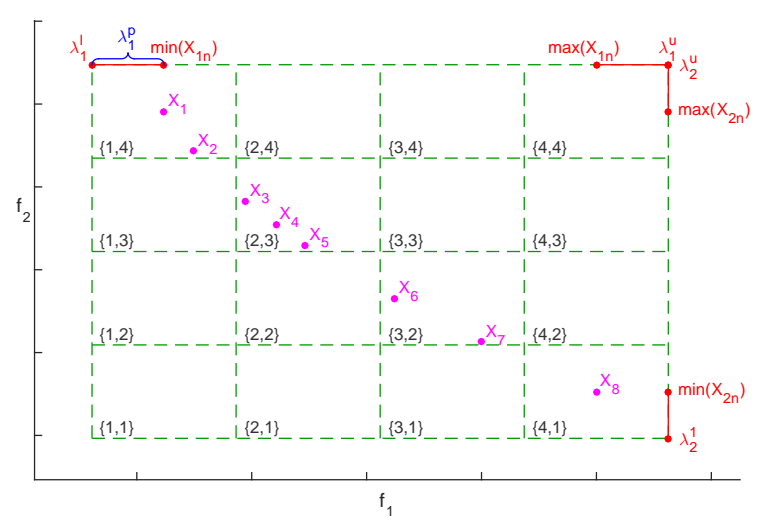

Figure 2. Visualisation of the AGA and its elements on a two-objective problem with four grid divisions. Vectors within braces indicate the grid location.

$$
\begin{aligned}
\lambda_{m}^{p} & =\frac{\left|\min \left(X_{m}\right)-\max \left(X_{m}\right)\right|}{2(\Delta-1)} \\
\lambda_{m}^{l} & =\min \left(X_{m}\right)-\lambda_{m}^{p} \\
\lambda_{m}^{u} & =\max \left(X_{m}\right)+\lambda_{m}^{p} \\
\lambda_{m} & =\left|\lambda_{m}^{l}-\lambda_{m}^{u}\right|
\end{aligned}
$$

where $\lambda_{m}^{l}$ is the start point of the grid for objective $m$ in the objective space, and $\lambda_{m}^{u}$ is the end point of the grid for objective $m$ in the objective space. These elements have been illustrated in Figure 2.

With the grid length and range calculated, it is possible to get the grid location of each solution's objective value using:

$$
\gamma_{m n}=\left\lceil\frac{x_{m n}-\lambda_{m}^{l}}{\frac{\lambda_{m}}{\Delta}}\right\rceil
$$

When the entries of $\Gamma_{n}$ have been calculated, it can be used to identify the grid location of a solution $X_{n}$. In this new method, the grid location $\Gamma_{n}$ is defined by a vector rather than a scalar, for example in a fiveobjective problem a grid location can be described by being at location $\Gamma_{n}=\langle 2,4,1,1,2\rangle$.

As an example, a population $X$ of five $(N=5)$ solutions $X_{n}$ for a five-objective problem $(M=5)$ has been presented in Table 1 .

The population $X$ has been subjected to the HAGA mechanism to resolve the grid location $\Gamma_{n}$ vec- 
Table 1. An example population $X$ of objective values $x_{m n}$ to be subjected to the HAGA mechanism.

\begin{tabular}{c|ccccc} 
& $x_{1 n}$ & $x_{2 n}$ & $x_{3 n}$ & $x_{4 n}$ & $x_{5 n}$ \\
\hline$X_{1}$ & 0.5 & 0.5 & 5.0 & 2.5 & 1.5 \\
$X_{2}$ & 0.6 & 0 & 5.0 & 3.0 & 1.4 \\
$X_{3}$ & 0.5 & 3.5 & 4.5 & 2.5 & 1.5 \\
$X_{4}$ & 0.8 & 3.2 & 4.2 & 3.0 & 1.2 \\
$X_{5}$ & 1 & 3 & 4 & 2 & 1
\end{tabular}

tor of each solution $X_{n}$, with an adaptive-grid configuration of four grid divisions $(\Delta=4)$. The grid locations resolved by the HAGA mechanism have been presented in Table 2. In this example, each solution $X_{n}$ has been assigned to a grid location $\Gamma_{n}$, for example, solution $X_{2}$ which consists of the objectives $\langle 0.5,0.5,5.0,2.5,1.5\rangle$ has been assigned to the grid location $\langle 2,1,4,4,3\rangle$. The solution values for each objective $x_{m n}$ have been plotted in their respective grid locations $\gamma_{m}$ in Figure 3.

Table 2. Grid locations $\Gamma$ for the example population $X$ of objective values $x_{m n}$.

\begin{tabular}{c|ccccc} 
& $\gamma_{1 n}$ & $\gamma_{2 n}$ & $\gamma_{3 n}$ & $\gamma_{4 n}$ & $\gamma_{5 n}$ \\
\hline$\Gamma_{1}$ & 1 & 1 & 4 & 3 & 4 \\
$\Gamma_{2}$ & 2 & 1 & 4 & 4 & 3 \\
$\Gamma_{3}$ & 1 & 4 & 2 & 3 & 4 \\
$\Gamma_{4}$ & 3 & 4 & 2 & 4 & 2 \\
$\Gamma_{5}$ & 4 & 4 & 1 & 1 & 1
\end{tabular}

The results from this example show that the example population does not consist of any solutions which are in the same grid square (otherwise their $\Gamma$ entries would be identical).

The method for selecting a grid location to remove a solution when the archive is at capacity is important when moving to many-objective problems. Selecting at random from grid locations which are at the same population density increases the probability of causing genetic drift, and decreases the diversity quality of the population. This undesirable effect is scaled exponentially as the number of objectives increase, and it is for this reason that many modern EMO algorithms now incorporate a niching approach when concerned with selection $[19,96]$. Therefore, it is desirable to find the grid location which is close to both solutions in the objective space and also at a higher density.
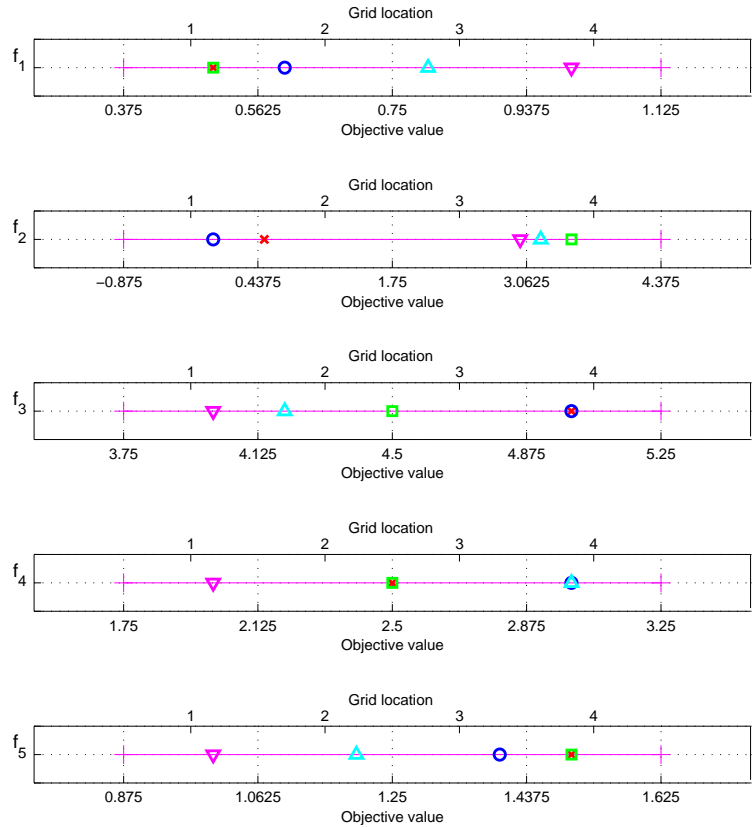

Figure 3. One dimensional plots illustrating the grid locations resolved by the HAGA mechanism for each objective value.

Storing grid locations as a single scalar value is not helpful when calculating distance between grid locations or for storing grid locations for a manyobjective problem. The grid location structure used in the proposed AGA scheme described above enables an intuitive method for finding the distance between grid locations. By establishing the grid location which a candidate solution would be assigned to if it was part of the archive, it is possible to find the difference between its grid location and other grid locations which are at high density to find out which one it's closest to by summing the difference of the grid location vectors.

For example, if a new solution

$$
X_{6}=\langle 0.6,0.5,4,3,1.1\rangle
$$

was to be included as a candidate solution as part of the HAGA mechanism, it would resolve a grid location of $\Gamma_{6}=\langle 2,1,1,4,2\rangle$. The distance $\delta_{n}$ between this grid location and the grid locations of the other solutions can be found by finding the absolute difference of each corresponding entry of the candidate solution's grid location and another solution from the population, and then summing those values. 


$$
\delta_{n}=\sum_{n=1}^{N}\left|\gamma_{m}^{*}-\gamma_{m n}\right|
$$

The distances $\delta_{n}$ between the grid location $\Gamma_{6}$ of solution $X_{6}$ and all the other solutions in the population presented in Table 1 have been presented in Table 3. The results show that the solution closest in proximity to solution $X_{6}$ is solution $X_{2}$.

Table 3. Grid locations $\Gamma$ for the example population $X$ of objective values $x_{m n}$.

\begin{tabular}{c|ccccc} 
& $\Gamma_{1}$ & $\Gamma_{2}$ & $\Gamma_{3}$ & $\Gamma_{4}$ & $\Gamma_{5}$ \\
\hline$\delta_{6}$ & 7 & 4 & 8 & 5 & 9
\end{tabular}

Using this method of resolving densely populated grid locations, which are closest in proximity to a candidate solution, it is possible to implement the approach listed in Algorithm 5.

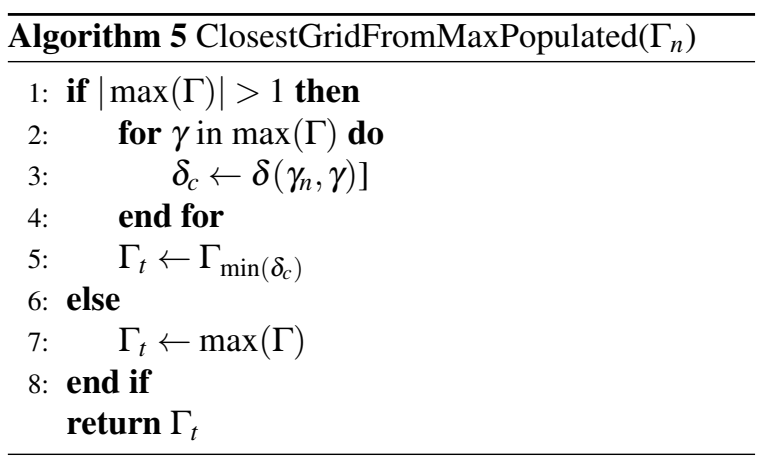

This algorithm is invoked by Line 5 of Algorithm 3 by Closest GridFromMaximallyPopulated $\left(X_{n}\right)$. Line 1 checks to see if there is a single grid location which contains the most solutions, if there is more than one grid location that contains the most number of solutions then the algorithm identifies the grid square which is closest in proximity to the candidate solution. $\delta_{n c}$ refers to the difference between the candidate solution's grid location and other grid locations, and $\Gamma_{\min \left(\delta_{c}\right)}$ refers to the grid location which is the closest in proximity among those containing the maximal number of solutions.

In previous AGA implementations, the extreme values for each objective were preserved at grid level. In the proposed AGA scheme, solutions containing ex- treme values for objectives (with the candidate solution taken into consideration) are removed from the population before it is subjected to the AGA. No special treatment is given to solutions containing locally (within their grid location) extreme objective values. This ensures candidate solutions are given a better chance of entering the archive than they would have had if they had come up against those solutions containing extreme values. This preserves the overall spread whilst encouraging new solutions to enter the archive.

\section{Numerical Results}

In order to test the proposed CMA-PAES-HAGA scalable WFG multi-objective test suite proposed in [32] has been considered in five, seven and ten objectives where each objective had 24 variables.

The proposed CMA-PAES-HAGA has been run with a parent population of size $\mu=100$ individuals, archive capacity of 100 individuals, number of grid divisions equal to 3 . The chosen value of 3 has been set subsequent to a tuning where the values between 2 and 20 have been taken into consideration. The 3 setting has been chosen as it proved to give the best performance in terms of computational cost. A lower value of grid divisions would result in fewer grid squares and therefore larger grid square populations. This will result in worst performance in the computation of the $\mathrm{CHV}$ indicator which is used at grid level. In contrast, higher numbers of grid divisions will result in many more grid locations which may hold only a few solutions and often only a single solution. This scenario does not ensure the best performance out of the $\mathrm{CHV}$ indicator, as often only a single solution will be in each grid square. The CMA-PAES-HAGA algorithm has been compared with MOEA/D-DRA [96] with both $\mu$ and $\lambda$ populations of 100 individuals, niche size of 20, and maximum update number equal to 2. A population size of 100 , in combination with the number of grid divisions set to 3 , prevents the computationally infeasible scenario where a large number of solutions are considered when calculating the hypervolume indicator values. Both algorithms considered in the pairwise comparisons use diversity operators in order to offer a well-distributed representation 
of the trade-off surface for each problem, with CMAPAES-HAGA using the hypervolume indicator driven approach, and MOEA/D-DRA using the weighted approach. Both the algorithms have been run for 50,000 function evaluations. Each algorithm for each problem has been run 30 times. The choice of this algorithm has been made considering that this is currently one of the algorithms which displayed the best performance on five-objective problems, see [70].

All test cases have been executed on a desktop computer with the configuration listed in Table 4.

Table 4. Hardware and software configurations of the computer used to generate the results.

\begin{tabular}{l|l} 
Configuration name & Configuration value \\
\hline Architecture & Linux-x64 \\
RAM & 16 GB \\
CPU & Intel(R) Xeon(R) CPU E5-1620 v2 \\
& $@ 3.70 \mathrm{GHz}$ \\
Total CPU Cores & 4 \\
MATLAB version & R2014a 64-bit (glnxa64) \\
Hypervolume Indicator & WFG HV 1.0.3
\end{tabular}

In order to evaluate the performance the hypervolume indicator has been chosen by following the indications in $[31,83]$. The hypervolume indicator is selected because it is scaling independent and requires no prior knowledge of the true Pareto-optimal front, this is important when working with real-world problems which have not yet been solved. The hypervolume indicator allows for a measurement as to which algorithm covers the greatest amount of the search space. A greater hypervolume indicator value indicates superior performance.

Tables 5, 6, and 7 show the hypervolume indicator on average and in the best and worst cases over the 30 runs available in the cases of five, seven and ten objectives, respectively. The best results are indicated in bold for clarity. The hypervolume indicator values can only be compared within a specific test-case for each of the considered algorithms. For example, the results for CMA-PAES-HAGA on the five-objective WFG1 test-case are directly comparable to the corresponding MOEA/D-DRA results on the same row of Table 5. This is because each test case requires a reference point, which is formed by using the extreme values encountered for each of the objectives in all of the 60 ap- proximation sets (30 runs for CMA-PAES-HAGA and 30 runs for MOEA/D-DRA).

In order to enhance the statistical significance of the results Wilcoxon test [89] has been performed. In each table, the $p$ value as well as the result of the Wilcoxon tests are shown: a "+" indicates that the proposed CMA-PAES-HAGA statistically outperformed MOEA/D-DRA, a "-" indicates that the proposed MOEA/D-DRA statistically outperformed CMA-PAES-HAGA, while a "=" indicates that the algorithm statistically have the same performance.

Table 5. Hypervolume results from 30 executions of CMA-PAESHAGA and MOEA/D-DRA on the WFG test suite with five objectives. The boldface values indicate superior performance

\begin{tabular}{lllllll|l|l}
\hline \multicolumn{1}{c}{ CMA-PAES-HAGA } & \multicolumn{3}{c}{ MOEA/D-DRA } \\
\hline & Worst & Mean & Best & Worst & Mean & Best & $p$-value & \\
\hline WFG1 & $\mathbf{0 . 4 1 3 9}$ & $\mathbf{0 . 4 1 6 3}$ & $\mathbf{0 . 4 1 9 4}$ & 0.3794 & 0.3906 & 0.397 & $3.019 \mathrm{e}-11$ & + \\
WFG2 & $\mathbf{0 . 8 5 0 6}$ & 0.8900 & 0.9041 & 0.7602 & $\mathbf{0 . 8 9 2 0}$ & $\mathbf{0 . 9 3 2 4}$ & 0.001302 & - \\
WFG3 & $\mathbf{0 . 5 9 4 0}$ & $\mathbf{0 . 6 1 9 4}$ & $\mathbf{0 . 6 3 9 8}$ & 0.5464 & 0.5843 & 0.6129 & $2.438 \mathrm{e}-09$ & + \\
WFG4 & $\mathbf{0 . 6 9 8 4}$ & $\mathbf{0 . 7 1 7 5}$ & $\mathbf{0 . 7 4 1 5}$ & 0.6109 & 0.6534 & 0.7056 & $4.077 \mathrm{e}-11$ & + \\
WFG5 & 0.52795 & 0.5638 & 0.5866 & $\mathbf{0 . 5 7 7 9}$ & $\mathbf{0 . 6 0 5 1}$ & $\mathbf{0 . 6 5 0 1}$ & $6.066 \mathrm{e}-11$ & - \\
WFG6 & $\mathbf{0 . 7 4 4 9}$ & $\mathbf{0 . 7 5 2 9}$ & $\mathbf{0 . 7 6 2 0}$ & 0.6746 & 0.7001 & 0.7219 & $3.019 \mathrm{e}-11$ & + \\
WFG7 & $\mathbf{0 . 6 1 3 0}$ & 0.6430 & 0.6756 & 0.5953 & $\mathbf{0 . 6 4 8 7}$ & $\mathbf{0 . 7 0 9 1}$ & 0.7172 & $=$ \\
WFG8 & $\mathbf{0 . 5 8 9 6}$ & $\mathbf{0 . 6 2 9 3}$ & 0.6555 & 0.5700 & 0.6098 & $\mathbf{0 . 6 6 9 7}$ & 0.0002 & + \\
WFG9 & $\mathbf{0 . 6 3 3 1}$ & $\mathbf{0 . 6 5 4 8}$ & $\mathbf{0 . 6 7 6 7}$ & 0.5359 & 0.5901 & 0.6339 & $3.338 \mathrm{e}-11$ & + \\
\hline \multicolumn{10}{c}{}
\end{tabular}

Table 6. Hypervolume results from 30 executions of CMA-PAESHAGA and MOEA/D-DRA on the WFG test suite with seven objectives. The boldface values indicate superior performance

\begin{tabular}{lllllll|l|l}
\hline \multicolumn{1}{c}{ CMA-PAES-HAGA } & \multicolumn{7}{c}{ MOEA/D-DRA } \\
\hline & Worst & Mean & Best & Worst & Mean & Best & $p$-value & \\
\hline WFG1 & $\mathbf{0 . 3 4 7 2}$ & $\mathbf{0 . 3 5 0 9}$ & $\mathbf{0 . 3 5 5 2}$ & 0.3191 & 0.3261 & 0.3348 & $3.019 \mathrm{e}-11$ & + \\
WFG2 & $\mathbf{0 . 8 5 5 5}$ & 0.8999 & 0.9272 & 0.7817 & $\mathbf{0 . 9 2 8 5}$ & $\mathbf{0 . 9 5 9 8}$ & $2.01 \mathrm{e}-08$ & - \\
WFG3 & $\mathbf{0 . 5 8 3 5}$ & $\mathbf{0 . 6 0 4 8}$ & $\mathbf{0 . 6 1 5 4}$ & 0.4917 & 0.5513 & 0.5936 & $4.0 \mathrm{e}-11$ & + \\
WFG4 & $\mathbf{0 . 6 8 3 4}$ & $\mathbf{0 . 7 0 5 6}$ & $\mathbf{0 . 7 2 4 7}$ & 0.5881 & 0.6509 & 0.7067 & $6.1 \mathrm{e}-10$ & + \\
WFG5 & 0.4755 & $\mathbf{0 . 5 2 8 1}$ & 0.5605 & $\mathbf{0 . 4 7 7 5}$ & 0.5253 & $\mathbf{0 . 5 8 2 3}$ & 0.3710 & $=$ \\
WFG6 & $\mathbf{0 . 8 4 8 4}$ & $\mathbf{0 . 8 5 4 6}$ & $\mathbf{0 . 8 5 9 2}$ & 0.8216 & 0.8326 & 0.8472 & $3.0 \mathrm{e}-11$ & + \\
WFG7 & $\mathbf{0 . 6 3 4 2}$ & $\mathbf{0 . 6 8 2 9}$ & 0.7222 & 0.5957 & 0.6634 & $\mathbf{0 . 7 3 9 2}$ & 0.0127 & + \\
WFG8 & $\mathbf{0 . 6 2 4 5}$ & $\mathbf{0 . 6 7 7 1}$ & 0.7188 & 0.6013 & 0.6671 & $\mathbf{0 . 7 3 3 5}$ & 0.5011 & $=$ \\
WFG9 & $\mathbf{0 . 5 7 8 6}$ & $\mathbf{0 . 6 2 3 1}$ & $\mathbf{0 . 6 5 3 2}$ & 0.4302 & 0.5228 & 0.6312 & $1.95 \mathrm{e}-10$ & + \\
\hline
\end{tabular}

Table 7. Hypervolume results from 30 executions of CMA-PAESHAGA and MOEA/D-DRA on the WFG test suite with ten objectives. The boldface values indicate superior performance.

\begin{tabular}{lllllll|l|l}
\hline \multicolumn{1}{c}{ CMA-PAES-HAGA } & \multicolumn{3}{c}{ MOEA/D-DRA } \\
\hline & Worst & Mean & Best & Worst & Mean & Best & $p$-value & \\
\hline WFG1 & $\mathbf{0 . 2 9 9 8}$ & $\mathbf{0 . 3 0 4 5}$ & $\mathbf{0 . 3 1 1 2}$ & 0.2713 & 0.2789 & 0.2910 & $3.019 \mathrm{e}-11$ & + \\
WFG2 & 0.9397 & 0.9559 & 0.9656 & $\mathbf{0 . 9 5 1 6}$ & $\mathbf{0 . 9 7 1 9}$ & $\mathbf{0 . 9 7 8 6}$ & $5.072 \mathrm{e}-10$ & - \\
WFG3 & $\mathbf{0 . 6 5 4 5}$ & $\mathbf{0 . 6 6 0 9}$ & $\mathbf{0 . 6 6 7 3}$ & 0.5315 & 0.5896 & 0.6260 & $3.012 \mathrm{e}-11$ & + \\
WFG4 & $\mathbf{0 . 6 7 4 4}$ & $\mathbf{0 . 7 0 7 5}$ & $\mathbf{0 . 7 6 7 9}$ & 0.6189 & 0.6990 & 0.765 & 0.3871 & $=$ \\
WFG5 & $\mathbf{0 . 4 6 5 8}$ & $\mathbf{0 . 4 9 2 7}$ & $\mathbf{0 . 5 1 2 7}$ & 0.4064 & 0.4522 & 0.5040 & $1.856 \mathrm{e}-09$ & + \\
WFG6 & $\mathbf{0 . 6 3 1 4}$ & $\mathbf{0 . 6 4 3 0}$ & $\mathbf{0 . 6 6 7 9}$ & 0.5458 & 0.5694 & 0.5981 & $3.019 \mathrm{e}-11$ & + \\
WFG7 & $\mathbf{0 . 6 7 2 7}$ & $\mathbf{0 . 7 2 5 0}$ & $\mathbf{0 . 8 0 5 1}$ & 0.5841 & 0.6627 & 0.7738 & $2.377 \mathrm{e}-07$ & + \\
WFG8 & $\mathbf{0 . 5 7 4 2}$ & $\mathbf{0 . 5 9 8 8}$ & 0.6341 & 0.4698 & 0.5827 & $\mathbf{0 . 6 8 1 3}$ & 0.3112 & $=$ \\
WFG9 & $\mathbf{0 . 5 4 4 4}$ & $\mathbf{0 . 6 0 4 6}$ & $\mathbf{0 . 6 3 4 8}$ & 0.3673 & 0.4414 & 0.5574 & $3.689 \mathrm{e}-11$ & + \\
\hline
\end{tabular}


It must be remarked that the calculation of the hypervolume indicator is, albeit a very reliable measure, computationally extremely onerous when more than three objectives are considered [6]. The calculation of a single hypervolume indicator value for a ten-objective approximation set can in some cases, require up to twelve hours with the hardware configuration specified in Table 4. For this reason we had to limit this comparison to only two algorithms. On the other hand we know from the literature, see e.g. [96], that MOEA/D-DRA outperforms many popular multiobjective optimisation algorithms on this test-suite.

Numerical results show that in the five-objective case, CMA-PAES-HAGA outperforms MOEA/D-DRA since the proposed algorithm displays a better performance for six problems and is outperformed in two cases. In the seven and ten objective cases, the performance of CMA-PAES-HAGA is clearly superior to that of MOEA/D-DRA. The proposed CMA-PAESHAGA significantly outperforms MOEA/D-DRA for six problems and is outperformed in only one case, that is WFG2 where the performance of MOEA/DDRA appears to be quite good. Although there is no general rigorous proof for the No Free Lunch Theorem for multi-objective optimisation $[17,90]$ the performance of multi-objective algorithms is also problem dependent.

Fig. 4, 5, and 6, show the variation in the hypervolume value of the algorithmic generations in five, seven, and ten objectives, respectively, in the case of WFG9. In each figure the hypervolume values associated to individual and average runs are highlighted.

Results in the figures clearly show how the performance of the proposed CMA-PAES-HAGA improves with the increase of the number of objectives with respect to the performance of MOEA/D-DRA. This trend is evident for WFG9 but is also generally true for the other test problems.

\subsection{Multi-objective Optimisation of Aircraft Control System Design}

An important engineering problem, here used as an application example, is the design of a fighter aircraft control system. This, as well as many engineering problems, are naturally multi-objective as engineers desire that multiple objectives are simultaneously op-

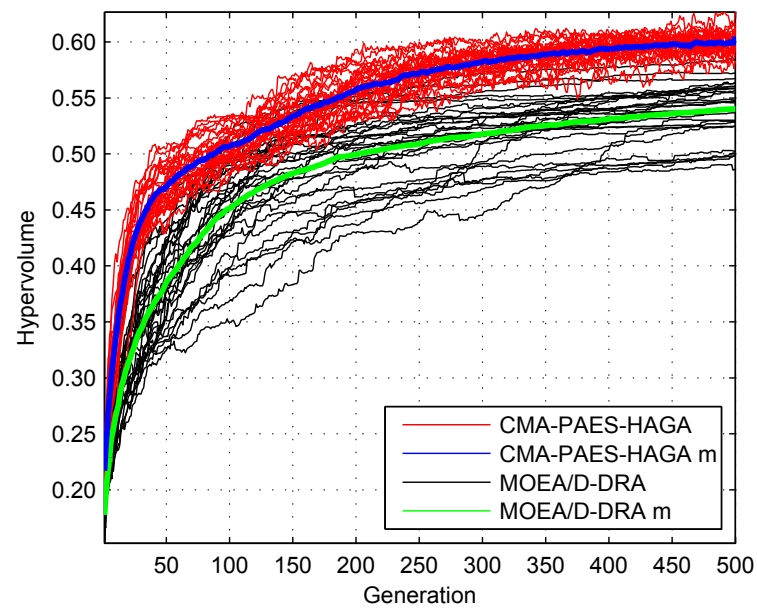

Figure 4. Evolution of the hypervolume indicator results (single runs and mean values $\mathrm{m}$ ) for five-objective WFG9 test case.

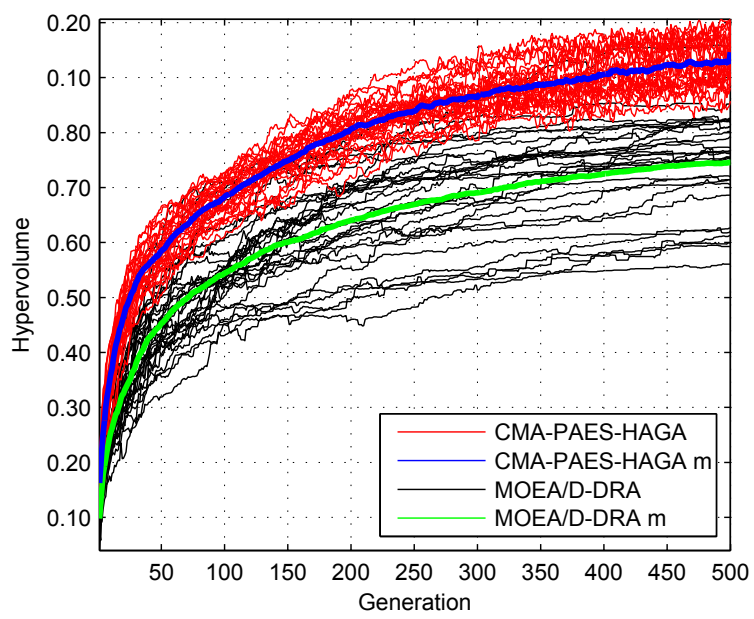

Figure 5. Evolution of the hypervolume indicator results (single runs and mean values $\mathrm{m}$ ) for seven-objective WFG9 test case.

timised. Figure 7 presents an illustration of an aircraft with the three main axes of motion labelled: the Roll (longitudinal) axis, the Pitch (lateral) axis, and the Yaw (vertical) axis. A combination of changes are made to both the angles and the rates of the angular velocities during the motion of the aircraft [80].

The system and input data for the fighter aircraft in this problem have been listed in the following: 


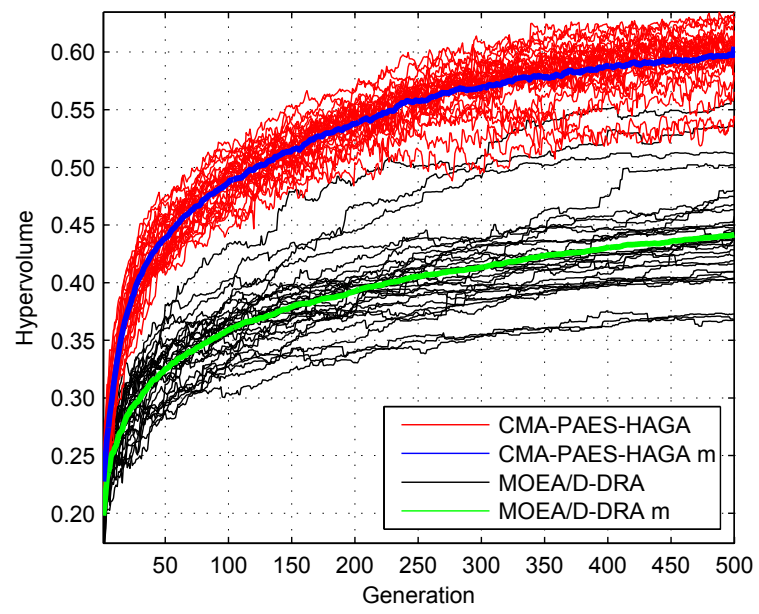

Figure 6. Evolution of the hypervolume indicator results (single runs and mean values $\mathrm{m}$ ) for ten-objective WFG9 test case.

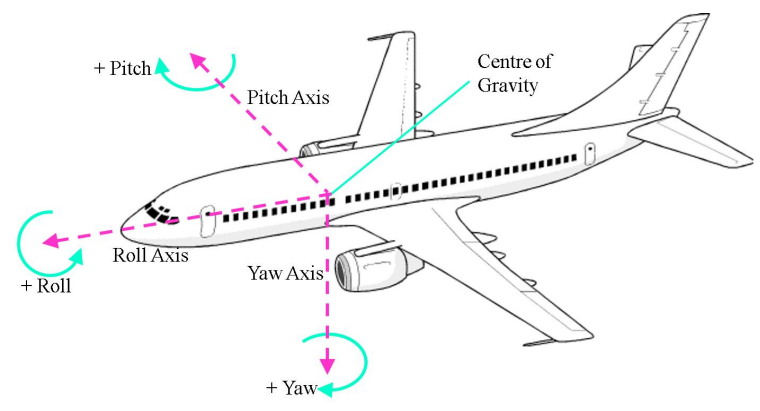

Figure 7. The three main axes of an Aircraft body.

$$
\begin{gathered}
\mathbf{A}=\left[\begin{array}{cccc}
-0.2842 & -0.9879 & 0.1547 & 0.0204 \\
10.8574 & -0.5504 & -0.2896 & 0.0000 \\
-199.8942 & -0.4840 & -1.6025 & 0.0000 \\
0.0000 & 0.1566 & 1.0000 & 0.0000
\end{array}\right] \\
\mathbf{B}=\left[\begin{array}{cc}
0.0000 & 0.0524 \\
0.4198 & -12.7393 \\
50.5756 & 21.6753 \\
0.0000 & 0.0000
\end{array}\right]
\end{gathered}
$$

These matrices represent the system state of the vehicle and are used in the control vector. More specifically, matrix $\mathbf{A}$ is the kinetic energy matrix while $\mathbf{B}$ is the Coriolis matrix, see [80] for details. Then,

$$
\mathbf{u}=\mathbf{C} \mathbf{u}_{\mathbf{p}}+\mathbf{K} \mathbf{x}
$$

where $\mathbf{u}_{\mathbf{p}}$ is the pilot's control input vector: $[16,0]$.

The seven problem variables $k_{1}$ to $k_{7}$ form part of the state space gain matrices, these represent gains applied to the various signals involved in the fighter aircraft control system:

$$
\mathbf{C}=\left[\begin{array}{cc}
1 & 0 \\
k_{5} & 1
\end{array}\right] ; \mathbf{K}=\left[\begin{array}{llll}
k_{6} & k_{1} & k_{2} & 0 \\
k_{7} & k_{3} & k_{4} & 0
\end{array}\right] .
$$

The optimisation problem studied in this example consists of finding the values of the gain coefficients $\left[k_{1}, k_{2}, k_{3}, k_{4}, k_{5}, k_{6}, k_{7}\right]$ such that the following seven objectives are simultaneously minimised:

1. The spiral root.

2. The damping in roll root.

3. The dutch-roll damping ratio.

4. The dutch-roll frequency.

5. The bank angle at 1.0 seconds.

6. The bank angle at 2.8 seconds.

7. The control effort.

Objectives 1 to 4 are the eigenvalues associated to the matrix $\mathbf{A}+\mathbf{B K}$, Objectives 5 and 6 are the bank angle taken at two intervals ( $1 \mathrm{~s}$ and $2.8 \mathrm{~s}$ ) according to Military Specification [8] requirements, Objective 7 is the sum of squares of gain vector.

Further details regarding the aircraft dynamic model and the problem variables are available in $[4,23]$. This optimisation problem will be referred to as Lateral Controller Synthesis (LATCON) herein.

The proposed CMA-PAES-HAGA has been tested to solve the LATCON problem and its performance has been compared with that of MOEA/D-DRA, NSGA-II, and a newly proposed algorithm for solving many-objective problems namely $\theta$-Dominance based Evolutionary Algorithm ( $\theta$-DEA) [92]. The parameter setting of CMA-PAES-HAGA and MOEA/D-DRA is the same mentioned above. NSGA-II was executed with $\mu$ and $\lambda$ populations of 100 individuals, and a mutation rate of $\frac{1}{n}$ where $n$ is the number of problem variables (seven in this case). The same population size $\mu=100$ has been used also for $\theta$-DEA. Each algorithm was given a limit of 10,000 function evaluations. The three competing algorithms have been chosen by considering that MOEA/D-DRA and $\theta$-DEA are specialised algorithms for problems with many objectives, whilst NSGA-II is used here as a classic 
benchmark algorithm. For each algorithm in this study 30 independent runs have been performed.

The hypervolume indicator was measured at each generation throughout the optimisation process. At the end of the optimisation the following hypervolume indicator average values have been yielded: CMA-PAES-HAGA (3.5616e+37), MOEA/D-DRA $(2.9182 \mathrm{e}+37), \theta$-DEA $(2.3992 \mathrm{e}+37)$ and NSGA-II $(2.3536 \mathrm{e}+36)$. These results clearly show that NSGAII, albeit popular and efficient for two-objective and three-objective problems, does not offer good performance on this seven-objective problem as the corresponding hypervolume indicator value is one order of magnitude inferior than that associated to the other two algorithms.

The comparison between CMA-PAES-HAGA, MOEA/D-DRA, and $\theta$-DEA show that although the latter two are good algorithms in multi-objective optimisation for problems with more than three objectives, they are both outperformed by CMA-PAES-HAGA on this control engineering problem.

The evolution of the hypervolume indicator for the four algorithms under consideration has been plotted in Fig. 8.

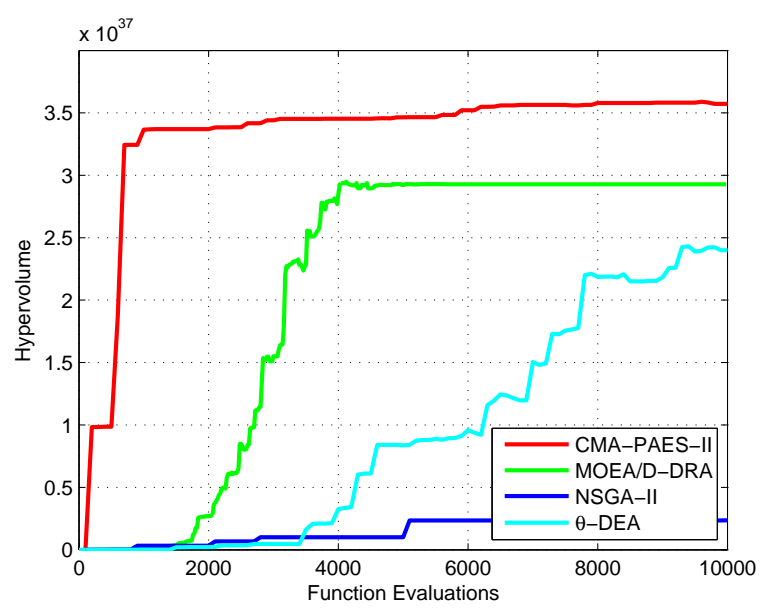

Figure 8. Evolution of the hypervolume indicator results for the seven-objective LATCON problem.

Fig. 8 highlights that NSGA-II is unsuitable to solve the LATCON problem. This result was expected as several studies show that NSGA-II displays poor performance in problems composed of more than three objectives, see e.g. [18]. In contrast, MOEA/D-DRA appears to be well suited to address the LATCON problem. This result was also expected as it confirms the findings reported in [38] where it is shown how a MOEA/D scheme tends to perform much better than NSGA-II in many-objective problems.

The performance of $\theta$-DEA is also quite good for this problem. However, $\theta$-DEA for this problem and for the given computational budget appears to display a performance which is not as good as that of CMAPAES-HAGA and MOEA/D-DRA. Furthermore, from an analysis of the trend in Fig. 8, unlike MOEA/DDRA, $\theta$-DEA probably required a larger budget to detect better values of hypervolume (the trend appears still to grow at the end of the budget).

The comparison between CMA-PAES-HAGA and MOEA/D-DRA indicates that the proposed algorithm not only achieves better hypervolume indicator values at the end of the computational budget, but also is much faster than MOEA/D-DRA to reach a good hypervolume indicator value. It can be observed that, for the LATCON problem, CMA-PAES-HAGA achieves a good hypervolume value after only 10 generations. This feature makes CMA-PAES-HAGA an interesting option for those problems which demand a solution in a short time or computationally expensive problems. Furthermore, it can be observed that at the end of the executions, while NSGA-II and MOEA/DDRA appear to be incapable to enhance upon their corresponding hypervolume indicator value, CMAPAES-HAGA is still able to obtain marginal improvements.

\section{Conclusion}

This article proposes a novel algorithm for multiobjective optimisation with four or more objectives. The proposed algorithm contains a novel selection mechanism suitable to compare solutions consisting of many objectives. This selection mechanism makes use of an approximated hypervolume logic in order to select solutions for the following generation. This selection mechanism overcomes the limitation of the true hypervolume indicator (selection by $\mathrm{CHV}$ ) whose application would be computationally infeasible, whilst still resulting in an algorithm which is very efficient for multi-objective problems with more than four objectives. 
Numerical results show that the proposed CMAPAES-HAGA framework displays an excellent performance in five, seven, and ten-objective optimisation problems when compared to MOEA/D-DRA which represents the state-of-the-art for many-objective optimisation problems. Furthermore, a real-world sevenobjective problem concerning the design of an aircraft control system is also used as a benchmark. In the latter case, it is evident that CMA-PAES-HAGA is a high-performance option, in terms of quickly achieving a high hypervolume indicator value, when the problem is characterised by many conflicting objectives.

\section{References}

[1] H. Adeli and K. C. Sarma, Cost Optimization of Structures: Fuzzy Logic, Genetic Algorithms, and Parallel Computing. New York, NY, USA: John Wiley \& Sons, Inc., 2006.

[2] M. Antonelli, P. Ducange, and F. Marcelloni, "A fast and efficient multi-objective evolutionary learning scheme for fuzzy rule-based classifiers," Information Sciences, vol. 283, no. 0 , pp. $36-54,2014$.

[3] J. Bader and E. Zitzler, "Hype: An algorithm for fast hypervolume-based many-objective optimization," Evolutionary Computation, vol. 19, no. 1, pp. 45-76, 2011.

[4] J. H. Blakelock, Automatic control of aircraft and missiles. John Wiley \& Sons, 1991.

[5] A. Bolourchi, S. F. Masri, and O. J. Aldraihem, "Studies into computational intelligence and evolutionary approaches for model-free identification of hysteretic systems," Computer-Aided Civil and Infrastructure Engineering, vol. 30, no. 5, pp. 330-346, 2015. [Online]. Available: http://dx.doi.org/10.1111/mice. 12126

[6] K. Bringmann, T. Friedrich, C. Igel, and T. Voß, "Speeding up many-objective optimization by monte carlo approximations," Artificial Intelligence, vol. 204, pp. 22-29, 2013.

[7] A. Cerveira, J. Baptista, and E. J. S. Pires, "Wind farm distribution network optimization," Integrated Computer-Aided Engineering, vol. 23, no. 1, pp. 69-79, 2015.

[8] C. R. Chalk, T. Neal, T. Harris, F. E. Pritchard, and R. J. Woodcock, "Background information and user guide for mil-f-8785b (asg),'military specification-flying qualities of piloted airplanes'," DTIC Document, Tech. Rep., 1969.

[9] J. Cheng, G. Zhang, F. Caraffini, and F. Neri, "Multicriteria adaptive differential evolution for global numerical optimization," Integrated Computer-Aided Engineering, vol. 22, no. 2, pp. 103-107, 2015.

[10] R. Cheng, Y. Jin, M. Olhofer, and B. Sendhoff, "A reference vector guided evolutionary algorithm for many-objective optimization," IEEE Transactions on Evolutionary Computation, vol. PP, no. 99, pp. 1-1, 2016, to appear.
[11] R. Cheng, M. Olhofer, and Y. Jin, "Reference vector based a posteriori preference articulation for evolutionary multiobjective optimization," in Evolutionary Computation (CEC), 2015 IEEE Congress on, May 2015, pp. 939-946.

[12] J. Y. J. Chow, "Activity-based travel scenario analysis with routing problem reoptimization," Computer-Aided Civil and Infrastructure Engineering, vol. 29, no. 2, pp. 91-106, 2014. [Online]. Available: http://dx.doi.org/10.1111/mice.12023

[13] C. A. C. Coello, "Evolutionary multi-objective optimization: some current research trends and topics that remain to be explored," Frontiers of Computer Science in China, vol. 3, no. 1, pp. 18-30, 2009.

[14] C. A. Coello Coello, "Research directions in evolutionary multi-objective optimization," Evolutionary Computation Journal, vol. 3, no. 3, pp. 110-121, 2012.

[15] C. A. Coello Coello and G. B. Lamont, Applications of multi-objective evolutionary algorithms. World Scientific, 2004, vol. 1 .

[16] L. Coletta, E. Hruschka, A. Acharya, and J. Ghosh, "Using metaheuristics to optimize the combination of classifier and cluster ensembles," Integrated Computer-Aided Engineering, vol. 22, no. 3, pp. 229-242, 2015.

[17] D. W. Corne and J. D. Knowles, "No free lunch and free leftovers theorems for multiobjective optimisation problems," in Evolutionary Multi-Criterion Optimization. Springer, 2003, pp. 327-341.

[18] K. Deb and H. Jain, "Handling many-objective problems using an improved nsga-ii procedure," in 2012 IEEE Congress on Evolutionary Computation, 2012, pp. 1-8.

[19] _ - "An evolutionary many-objective optimization algorithm using reference-point-based nondominated sorting approach, part i: Solving problems with box constraints," Evolutionary Computation, IEEE Transactions on, vol. 18, no. 4, pp. 577-601, Aug 2014.

[20] K. Deb, "Multi-objective optimization," Multi-objective optimization using evolutionary algorithms, pp. 13-46, 2001.

[21] K. Deb, A. Pratap, S. Agarwal, and T. Meyarivan, "A fast and elitist multiobjective genetic algorithm: Nsga-ii," Evolutionary Computation, IEEE Transactions on, vol. 6, no. 2, pp. 182-197, 2002.

[22] M. Emmerich, N. Beume, and B. Naujoks, "An emo algorithm using the hypervolume measure as selection criterion," in Evolutionary Multi-Criterion Optimization. Springer, 2005, pp. 62-76.

[23] B. Etkin, Dynamics of atmospheric flight. Courier Corporation, 2012.

[24] R. M. Everson, J. E. Fieldsend, and S. Singh, Adaptive Computing in Design and Manufacture $V$. London: Springer London, 2002, ch. Full Elite Sets for Multi-objective Optimisation, pp. 343-354.

[25] M. Farina and P. Amato, "On the optimal solution definition for many-criteria optimization problems," in Proceedings of the NAFIPS-FLINT International Conference, J. Keller and O. Nasraoui, Eds., 2002, pp. 233 - 238.

[26] M. Fleischer, Evolutionary Multi-Criterion Optimization: Second International Conference, EMO 2003, Faro, Portu- 
gal, April 8-11, 2003. Proceedings. Springer Berlin Heidelberg, 2003, ch. The Measure of Pareto Optima Applications to Multi-objective Metaheuristics, pp. 519-533.

[27] C. M. Fonseca and P. J. Fleming, "Multiobjective optimization and multiple constraint handling with evolutionary algorithms. i. a unified formulation," Systems, Man and Cybernetics, Part A: Systems and Humans, IEEE Transactions on, vol. 28, no. 1, pp. 26-37, 1998.

[28] C. M. Fonseca, L. Paquete, and M. López-Ibánez, "An improved dimension-sweep algorithm for the hypervolume indicator," in Evolutionary Computation, 2006. CEC 2006. IEEE Congress on. IEEE, 2006, pp. 1157-1163.

[29] A. Ghahari and J. D. Enderle, "A neuron-based time-optimal controller of horizontal saccadic eye movements," Int. J. Neural Syst., vol. 24, no. 6, 2014.

[30] N. Hansen and A. Ostermeier, "Completely derandomized self-adaptation in evolution strategies," Evolutionary computation, vol. 9, no. 2, pp. 159-195, 2001.

[31] M. Helbig and A. P. Engelbrecht, "Performance measures for dynamic multi-objective optimisation algorithms," Information Sciences, vol. 250, pp. 61-81, 2013.

[32] S. Huband, P. Hingston, L. Barone, and L. While, "A review of multiobjective test problems and a scalable test problem toolkit," Evolutionary Computation, IEEE Transactions on, vol. 10, no. 5, pp. 477-506, 2006.

[33] E. J. Hughes, "Evolutionary many-objective optimisation: many once or one many?" in Evolutionary Computation, 2005. The 2005 IEEE Congress on, vol. 1. IEEE, 2005, pp. 222-227.

[34] _ - "Msops-ii: A general-purpose many-objective optimiser," in Evolutionary Computation, 2007. CEC 2007. IEEE Congress on. IEEE, 2007, pp. 3944-3951.

[35] G. Iacca, F. Caraffini, and F. Neri, "Multi-strategy coevolving aging particle optimization," Int. J. Neural Syst., vol. 24, no. $1,2014$.

[36] C. Igel, N. Hansen, and S. Roth, "Covariance matrix adaptation for multi-objective optimization," Evolutionary computation, vol. 15, no. 1, pp. 1-28, 2007.

[37] H. Ishibuchi, N. Akedo, and Y. Nojima, "Behavior of multiobjective evolutionary algorithms on many-objective knapsack problems," IEEE Transactions on Evolutionary Computation, vol. 19, no. 2, pp. 264-283, April 2015.

[38] H. Ishibuchi, Y. Sakane, N. Tsukamoto, and Y. Nojima, "Evolutionary many-objective optimization by nsga-ii and moea/d with large populations," in Systems, Man and Cybernetics, 2009. SMC 2009. IEEE International Conference on, 2009, pp. 1758-1763.

[39] H. Ishibuchi, N. Tsukamoto, Y. Hitotsuyanagi, and Y. Nojima, "Effectiveness of scalability improvement attempts on the performance of nsga-ii for many-objective problems," in Proceedings of the 10th annual conference on Genetic and evolutionary computation. ACM, 2008, pp. 649-656.

[40] H. Ishibuchi, N. Tsukamoto, and Y. Nojima, "Evolutionary many-objective optimization: A short review." in IEEE Congress on Evolutionary Computation, 2008, pp. 24192426 .
[41] H. Ishibuchi, N. Tsukamoto, Y. Sakane, and Y. Nojima, "Indicator-based evolutionary algorithm with hypervolume approximation by achievement scalarizing functions," in Proceedings of the 12th annual conference on Genetic and evolutionary computation. ACM, 2010, pp. 527-534.

[42] H. Ishibuchi, T. Yoshida, and T. Murata, "Balance between Genetic Search and Local Search in Memetic Algorithms for Multiobjective permutation Flow shop Scheduling," IEEE Transactions on Evolutionary Computation, vol. 7 , no. 2, pp. 204-223, 2003.

[43] H. Jain and K. Deb, "An evolutionary many-objective optimization algorithm using reference-point based nondominated sorting approach, part ii: Handling constraints and extending to an adaptive approach," IEEE Transactions on Evolutionary Computation, vol. 18, no. 4, pp. 602-622, Aug 2014.

[44] A. Jaszkiewicz, "On the computational efficiency of multiple objective metaheuristics. the knapsack problem case study," European Journal of Operational Research, vol. 158, no. 2, pp. 418-433, 2004.

[45] L. Jia, Y. Wang, and L. Fan, "Multiobjective bilevel optimization for production-distribution planning problems using hybrid genetic algorithm," Integrated Computer-Aided Engineering, vol. 21, no. 1, pp. 77-90, 2014.

[46] Y. Jin and B. Sendhoff, "Connectedness, regularity and the success of local search in evolutionary multi-objective optimization," in Evolutionary Computation, 2003. CEC '03. The 2003 Congress on, vol. 3, Dec 2003, pp. 1910-1917.

[47] M. Joly, T. Verstraete, and G. Paniagua, "Integrated multifidelity, multidisciplinary evolutionary design optimization of counterrotating compressors," Integrated Computer-Aided Engineering, vol. 21, no. 3, pp. 249-261, 2014.

[48] V. Khare, X. Yao, and K. Deb, "Performance scaling of multi-objective evolutionary algorithms," in Evolutionary Multi-Criterion Optimization. Springer, 2003, pp. 376390

[49] H. Kim and H. Adeli, "Discrete cost optimization of composite floors using a floating-point genetic algorithm," Engineering Optimization, vol. 33, no. 4, pp. 485-501, 2001.

[50] J. D. Knowles, D. W. Corne, and M. Fleischer, "Bounded archiving using the lebesgue measure," in Evolutionary Computation, 2003. CEC '03. The 2003 Congress on, vol. 4 , Dec 2003, pp. 2490-2497 Vol.4.

[51] J. Knowles and D. Corne, "The pareto archived evolution strategy: A new baseline algorithm for pareto multiobjective optimisation," in Evolutionary Computation, 1999. CEC 99. Proceedings of the 1999 Congress on, vol. 1. IEEE, 1999.

[52] _ "Quantifying the effects of objective space dimension in evolutionary multiobjective optimization," in Proceedings of the 4th International Conference on Evolutionary Multi-criterion Optimization, ser. EMO'07. SpringerVerlag, 2007, pp. 757-771.

[53] M. Kociecki and H. Adeli, "Two-phase genetic algorithm for size optimization of free-form steel space-frame roof structures," Journal of Constructional Steel Research, vol. 90 , pp. 283 - 296, 2013. 
[54] _ _ "Two-phase genetic algorithm for topology optimization of free-form steel space-frame roof structures with complex curvatures," Engineering Applications of Artificial Intelligence, vol. 32, pp. 218 - 227, 2014.

[55] _ _ "Shape optimization of free-form steel space-frame roof structures with complex geometries using evolutionary computing," Eng. Appl. of AI, vol. 38, pp. 168-182, 2015.

[56] A. Lara, G. Sanchez, C. A. Coello Coello, and O. Schutze, "Hcs: a new local search strategy for memetic multiobjective evolutionary algorithms," Evolutionary Computation, IEEE Transactions on, vol. 14, no. 1, pp. 112-132, 2010.

[57] H.-G. Lee, C.-Y. Yi, D.-E. Lee, and D. Arditi, "An advanced stochastic time-cost tradeoff analysis based on a cpm-guided genetic algorithm," ComputerAided Civil and Infrastructure Engineering, vol. 30, no. 10, pp. 824-842, 2015. [Online]. Available: http://dx.doi.org/10.1111/mice. 12148

[58] B. Li, J. Li, K. Tang, and X. Yao, "Many-objective evolutionary algorithms: A survey," ACM Comput. Surv., vol. 48, no. 1, pp. 13:1-13:35, Sep. 2015.

[59] D.-Y. Lin and Y.-H. Ku, "Using genetic algorithms to optimize stopping patterns for passenger rail transportation," Computer-Aided Civil and Infrastructure Engineering, vol. 29, no. 4, pp. 264-278, 2014. [Online]. Available: http://dx.doi.org/10.1111/mice. 12020

[60] A. López-Jaimes and C. A. Coello Coello, "Including preferences into a multiobjective evolutionary algorithm to deal with many-objective engineering optimization problems," Information Sciences, vol. 277, pp. 1-20, 2014

[61] A. Lpez-Jaimes and C. A. C. Coello, "Including preferences into a multiobjective evolutionary algorithm to deal with many-objective engineering optimization problems," Information Sciences, vol. 277, no. 0, pp. 1 - 20, 2014.

[62] H. Menendez, D. Barrero, and D. Camacho, "A genetic graph-based approach to the partitional clustering," International Journal of Neural Systems, vol. 24, no. 3, 2015, 1430008.

[63] P. Mesejo, O. Ibanez Enrique Fernandez-Blanco, F. Cedron, A. Pazos, and A. Porto-Pazos, "Artificial neuron-glia networks learning paradigm based on cooperative coevolution," International Journal of Neural Systems, vol. 25, no. 4, 2015, 1550012.

[64] E. Mezura-Montes, M. Reyes-Sierra, and C. A. Coello Coello, "Multi-Objective Optimization using Differential Evolution: A Survey of the State-of-the-Art," in Advances in Differential Evolution, ser. Studies in Computational Intelligence, U. K. Chakraborty, Ed. Springer, 2008, vol. 143, pp. 173-196.

[65] K. Miettinen, Nonlinear multiobjective optimization. Springer, 1999, vol. 12.

[66] C. A. Perez-Ramirez, J. P. Amezquita-Sanchez, H. Adeli, M. Valtierra-Rodriguez, D. Camarena-Martinez, and R. de Jesús Romero-Troncoso, "New methodology for modal parameters identification of smart civil structures using ambient vibrations and synchrosqueezed wavelet transform," Eng. Appl. of AI, vol. 48, pp. 1-12, 2016.
[67] R. C. Purshouse, "On the evolutionary optimisation of many objectives," Ph.D. dissertation, Department of Automatic Control and Systems Engineering, University of Sheffield, Sheffield, UK, S1 3JD, 2003.

[68] R. C. Purshouse and P. J. Fleming, "On the evolutionary optimization of many conflicting objectives," Evolutionary Computation, IEEE Transactions on, vol. 11, no. 6, pp. 770784, 2007.

[69] S. Rashidi and P. Ranjitkar, "Bus dwell time modeling using gene expression programming," ComputerAided Civil and Infrastructure Engineering, vol. 30, no. 6, pp. 478-489, 2015. [Online]. Available: http://dx.doi.org/10.1111/mice.12125

[70] S. Rostami, "Preference focussed many-objective evolutionary computation," Ph.D. dissertation, School of Engineering, Manchester Metropolitan University, Manchester, UK, M15 6HB, 2014.

[71] S. Rostami, D. OReilly, A. Shenfield, and N. Bowring, "A novel preference articulation operator for the evolutionary multi-objective optimisation of classifiers in concealed weapons detection," Information Sciences, vol. 295, no. 0, pp. $494-520,2015$.

[72] S. Rostami and A. Shenfield, "Cma-paes: Pareto archived evolution strategy using covariance matrix adaptation for multi-objective optimisation," in Proceedings of the IEEE UK conference on Computational Intelligence (UKCI), Edinburgh, UK, September 2012, pp. 1-8.

[73] K. C. Sarma and H. Adeli, "Cost optimization of steel structures," Engineering Optimization, vol. 32, pp. 777-802, 2000.

[74] _ - "Fuzzy genetic algorithm for optimization of steel structures," Journal of Structural Engineering, vol. 126 , no. 5, pp. 596-604, 2000.

[75] F. Shabbir and P. Omenzetter, "Particle swarm optimization with sequential niche technique for dynamic finite element model updating," Computer-Aided Civil and Infrastructure Engineering, vol. 30, no. 5, pp. 359-375, 2015. [Online]. Available: http://dx.doi.org/10.1111/mice.12100

[76] S. Shapero, M. Zhu, J. Hasler, and C. J. Rozell, "Optimal sparse approximation with integrate and fire neurons," Int. J. Neural Syst., vol. 24, no. 5, 2014.

[77] N. Siddique and H. Adeli, Computational Intelligence: Synergies of Fuzzy Logic, Neural Networks and Evolutionary Computing. J. Whiley and sons, 2013.

[78] N. H. Siddique and H. Adeli, "Applications of harmony search algorithms in engineering," International Journal on Artificial Intelligence Tools, vol. 24, no. 6, 2015.

[79] K. Sindhya, K. Miettinen, and K. Deb, "A hybrid framework for evolutionary multi-objective optimization," IEEE Transactions on Evolutionary Computation, vol. 17, no. 4, pp. 495-511, Aug 2013.

[80] D. Tabak, A. Schy, D. Giesy, and K. Johnson, "Application of multiobjective optimization in aircraft control systems design," Automatica, vol. 15, no. 5, pp. 595-600, 1979.

[81] K. C. Tan, S. C. Chiam, A. A. Mamun, and C. K. Goh, "Balancing exploration and exploitation with adaptive vari- 
ation for evolutionary multi-objective optimization," European Journal of Operational Research, vol. 197, pp. 701713, 2009.

[82] K. C. Tan, E. F. Khor, and T. H. Lee, Multiobjective Evolutionary Algorithms and Applications: Algorithms and Applications. Springer, 2006.

[83] K. Van Moffaert, M. M. Drugan, and A. Nowé, "Hypervolume-based multi-objective reinforcement learning," in Evolutionary Multi-Criterion Optimization. Springer, 2013, pp. 352-366.

[84] L. Vincenzi and M. Savoia, "Coupling response surface and differential evolution for parameter identification problems," Computer-Aided Civil and Infrastructure Engineering, vol. 30, no. 5, pp. 376-393, 2015. [Online]. Available: http://dx.doi.org/10.1111/mice. 12124

[85] T. Voß, N. Hansen, and C. Igel, "Improved step size adaptation for the mo-cma-es," in Proceedings of the 12th annual conference on Genetic and evolutionary computation. ACM, 2010, pp. 487-494.

[86] D. J. Walker, R. Everson, and J. E. Fieldsend, "Visualizing mutually nondominating solution sets in many-objective optimization," IEEE Transactions on Evolutionary Computation, vol. 17, no. 2, pp. 165-184, April 2013.

[87] X. Wang, G. Zhang, F. Neri, T. Jiang, J. Zhao, M. Gheorghe, F. Ipate, and R. Lefticaru, "Design and implementation of membrane controllers for trajectory tracking of nonholonomic wheeled mobile robots," Integrated Computer-Aided Engineering, vol. 23, no. 1, pp. 15-30, 2015.

[88] L. While, P. Hingston, L. Barone, and S. Huband, "A faster algorithm for calculating hypervolume," Evolutionary Computation, IEEE Transactions on, vol. 10, no. 1, pp. 29-38, 2006.

[89] F. Wilcoxon, "Individual comparisons by ranking methods," Biometrics bulletin, pp. 80-83, 1945.

[90] D. H. Wolpert and W. G. Macready, "No free lunch theorems for optimization," Evolutionary Computation, IEEE Transactions on, vol. 1, no. 1, pp. 67-82, 1997.

[91] J. Wu and S. Azarm, "Metrics for quality assessment of a multiobjective design optimization solution set," ASME. $J$. Mech. Des., vol. 123, pp. 18-25, 2000.

[92] Y. Yuan, H. Xu, B. Wang, and X. Yao, "A new dominance relation-based evolutionary algorithm for many-objective optimization," IEEE Transactions on Evolutionary Computation, vol. 20, no. 1, pp. 16-37, Feb 2016.

[93] A. Zamuda, J. Brest, B. Boskovic, and V. Zumer, "Differential evolution for multiobjective optimization with self adaptation." in IEEE Congress on Evolutionary Computation, 2007, pp. 3617-3624.

[94] G. Zhang, H. Rong, F. Neri, and M. J. Pérez-Jiménez, “An optimization spiking neural $\mathrm{P}$ system for approximately solving combinatorial optimization problems," Int. J. Neural Syst., vol. 24, no. 5, 2014.

[95] Q. Zhang and H. Li, "Moea/d: A multiobjective evolutionary algorithm based on decomposition," Evolutionary Computation, IEEE Transactions on, vol. 11, no. 6, pp. 712-731, 2007.
[96] Q. Zhang, W. Liu, and H. Li, "The performance of a new version of moea/d on cec09 unconstrained mop test instances," in Evolutionary Computation, 2009. CEC'09. IEEE Congress on. IEEE, 2009, pp. 203-208.

[97] Q. Zhang and P. N. Suganthan, "Final report on cec09 moea competition," in Congress on evolutionary computation (CEC 2009), 2009.

[98] X. Zhang, Y. Tian, R. Cheng, and Y. Jin, "An efficient approach to nondominated sorting for evolutionary multiobjective optimization," IEEE Transactions on Evolutionary Computation, vol. 19, no. 2, pp. 201-213, April 2015.

[99] X. Zhang, Y. Tian, and Y. Jin, "A knee point-driven evolutionary algorithm for many-objective optimization," IEEE Transactions on Evolutionary Computation, vol. 19, no. 6, pp. 761-776, Dec 2015.

[100] A. Zhou, B.-Y. Qu, H. Li, S.-Z. Zhao, P. N. Suganthan, and Q. Zhang, "Multiobjective evolutionary algorithms: A survey of the state of the art," Swarm and Evolutionary Computation, vol. 1, no. 1, pp. 32-49, 2011.

[101] W. Zhu, H. Hu, and Z. Huang, "Calibrating rail transit assignment models with genetic algorithm and automated fare collection data," Computer-Aided Civil and Infrastructure Engineering, vol. 29, no. 7, pp. 518-530, 2014. [Online]. Available: http://dx.doi.org/10.1111/mice.12075

[102] E. Zitzler and S. Künzli, "Indicator-based selection in multiobjective search," in Parallel Problem Solving from NaturePPSN VIII. Springer, 2004, pp. 832-842.

[103] X. Zou, Y. Chen, M. Liu, and L. Kang, "A new evolutionary algorithm for solving many-objective optimization problems," Systems, Man, and Cybernetics, Part B: Cybernetics, IEEE Transactions on, vol. 38, no. 5, pp. 1402-1412, 2008. 